NISSUNA UMANA INVESTIGAZIONE SI PUO DIMANDARE VERA SCIENZIA S'ESSA NON PASSA PER LE MATEMATICHE DIMOSTRAZIONI LEONARDO DA VINCI

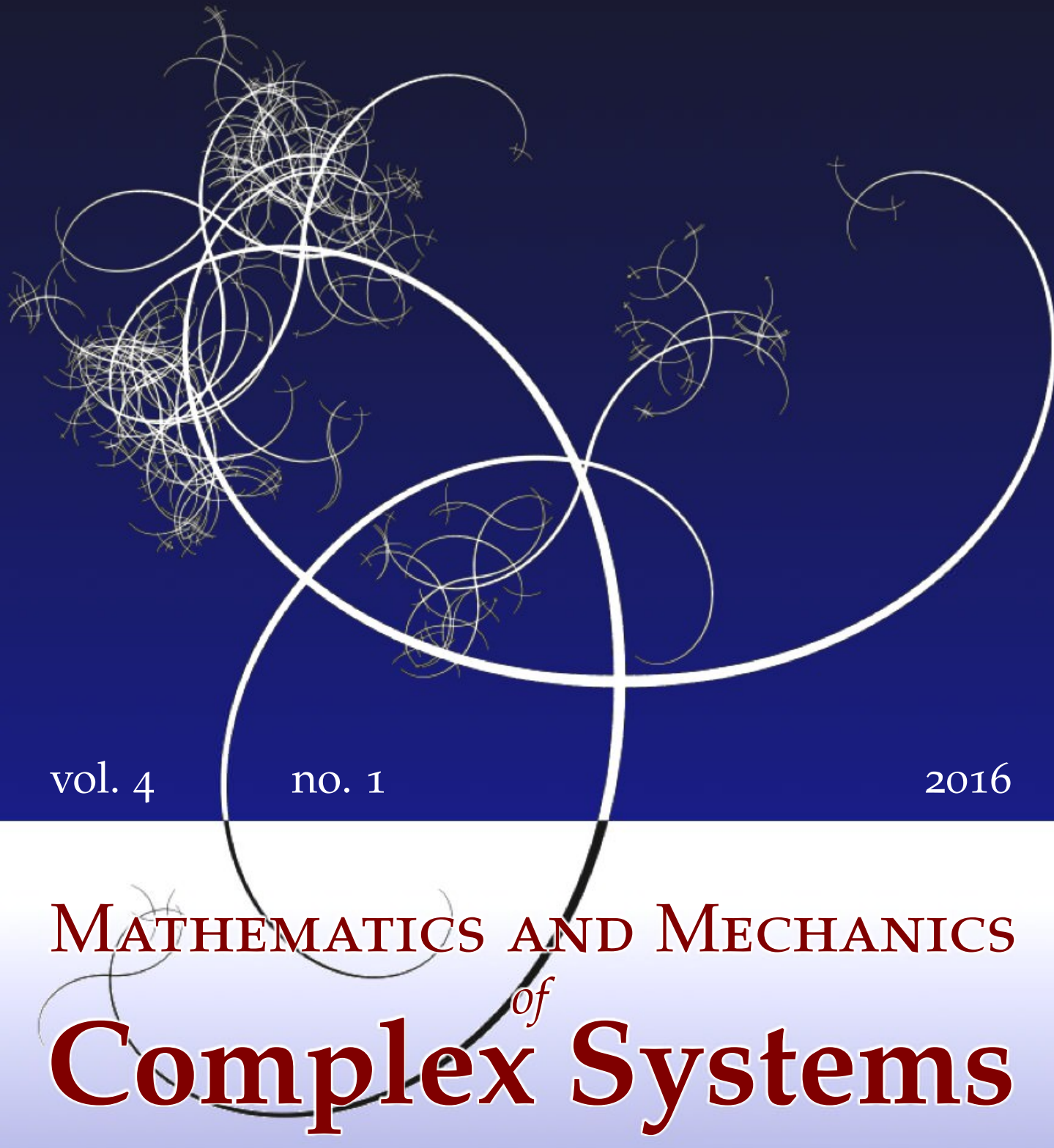

Andrea Braides, Valeria Chiadò Piat and Margherita Solci DISCRETE DOUBLE-POROSITY MODELS FOR SPIN SYSTEMS 


\title{
DISCRETE DOUBLE-POROSITY MODELS FOR SPIN SYSTEMS
}

\author{
Andrea Braides, VAleria Chiadò Piat And Margherita Solci
}

We consider spin systems between a finite number $N$ of "species" or "phases" partitioning a cubic lattice $\mathbb{Z}^{d}$. We suppose that interactions between points of the same phase are coercive while those between points of different phases (or possibly between points of an additional "weak phase") are of lower order. Following a discrete-to-continuum approach, we characterize the limit as a continuum energy defined on $N$-tuples of sets (corresponding to the $N$ strong phases) composed of a surface part, taking into account homogenization at the interface of each strong phase, and a bulk part that describes the combined effect of lowerorder terms, weak interactions between phases, and possible oscillations in the weak phase.

\section{Introduction}

In this paper, we consider lattice spin energies mixing strong ferromagnetic interactions and weak (possibly antiferromagnetic) pair interactions. The geometry that we have in mind is a periodic system of interactions such as that whose periodicity cell is represented in Figure 1. In that picture, the strong interactions between nodes of the lattice (circles) are represented by solid lines and weak ones by dashed lines. In this particular case, we have two three-periodic systems of "strong sites", i.e., sites connected by strong interactions, and isolated "weak sites" (pictured as white circles). Note that we may also have one or more infinite systems of connected weak interactions as in Figure 2. In a discrete environment, the topological requirements governing the interactions between the strong and weak phases characteristic of continuum high-contrast models are substituted with assumptions on long-range interactions. In particular, contrary to the continuum case, for discrete systems with second-neighbor (or longer-range) interactions, we may have a limit multiphase system even in dimension 1 (see the examples in Section 6).

This paper is part of a general study of spin systems by means of variational techniques through the computation of continuum approximate energies, for which

\section{Communicated by Raffaele Esposito.}

The authors are members of INdAM-GNAMPA.

MSC2010: 49M25, 39A12, 39A70, 35Q82.

Keywords: spin systems, lattice energies, double porosity, $\Gamma$-convergence, homogenization, discrete to continuum, high contrast, interfacial energies, multiphase materials. 


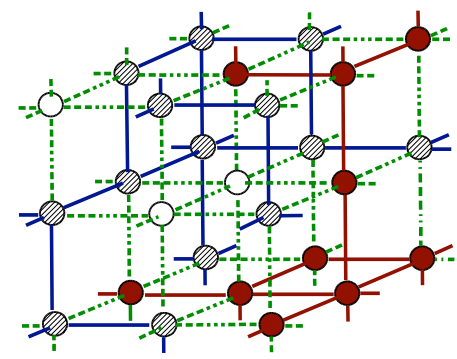

Figure 1. Picture of a double-porosity system.

homogenization results have been proved in the ferromagnetic case (i.e, when all interactions are strong) [Caffarelli and de la Llave 2005; Braides and Piatnitski 2013], and a general discrete-to-continuum theory of representation and optimization has been developed (see the survey [Braides 2014a]). In particular, a discreteto-continuum compactness result and an integral representation of the limit by means of surface energies defined on sets of finite perimeter have been proved [Alicandro and Gelli 2016]. In that result, the coercivity of energies is obtained by assuming that nearest neighbors are always connected through a chain of strong interactions. Double-porosity systems can be interpreted as energies for which this condition does not hold but is satisfied separately on (finitely many) infinite connected components.

We are going to consider energies defined on functions parametrized on the cubic lattice $\mathbb{Z}^{d}$ of the form

$$
\begin{aligned}
F_{\varepsilon}(u)= & \sum_{(\alpha, \beta) \in \varepsilon \mathcal{N}_{1} \cap(\Omega \times \Omega)} \varepsilon^{d-1} a_{\alpha \beta}^{\varepsilon}\left(u_{\alpha}-u_{\beta}\right)^{2} \\
+ & \sum_{(\alpha, \beta) \in \mathcal{E} \mathcal{N}_{0} \cap(\Omega \times \Omega)} \varepsilon^{d} a_{\alpha \beta}^{\varepsilon}\left(u_{\alpha}-u_{\beta}\right)^{2}+\sum_{\alpha \in \Omega \cap \varepsilon \mathbb{Z}^{d}} \varepsilon^{d} g\left(u_{\alpha}\right),
\end{aligned}
$$

where $\Omega$ is a regular open subset of $\mathbb{R}^{d}$ and $u_{\alpha} \in\{-1,+1\}$ denote the values of a spin function. For explanatory purposes, in this formula and the rest of the

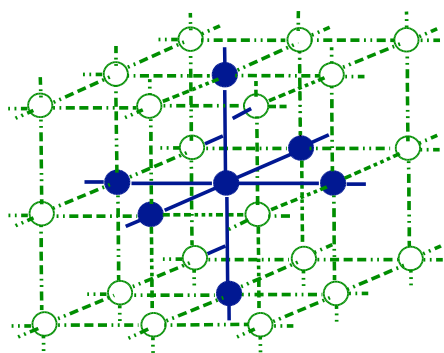

Figure 2. A double-porosity system with an infinite connected weak component. 
introduction, we use a simplified notation with respect to the rest of the paper, defining $u=\left\{u_{\alpha}\right\}$ on the nodes of $\Omega \cap \varepsilon \mathbb{Z}^{d}$ (instead of, equivalently, on the nodes of $\left.(1 / \varepsilon) \Omega \cap \mathbb{Z}^{d}\right)$. We denote by $\mathcal{N}_{1}$ the set of pairs of nodes in $\mathbb{Z}^{d} \times \mathbb{Z}^{d}$ between which we have strong interactions and by $\mathcal{N}_{0}$ the set of pairs in $\mathbb{Z}^{d} \times \mathbb{Z}^{d}$ between which we have weak interactions. The difference between these two types of interactions in the energy is the scaling factor: $\varepsilon^{d-1}$ for strong interactions and $\varepsilon^{d}$ for weak interaction. We suppose that all coefficients are obtained by scaling fixed coefficients on $\mathbb{Z}^{d}$, i.e.,

$$
a_{\alpha \beta}^{\varepsilon}=a_{\alpha / \varepsilon, \beta / \varepsilon} \quad \text { if } \alpha, \beta \in \varepsilon \mathbb{Z}^{d},
$$

and $a_{j k}$ are periodic of some integer period $T$. Moreover, we assume that the coefficients of the strong interactions are strictly positive, i.e., $a_{j k}>0$ if $(j, k) \in \mathcal{N}_{1}$. The "forcing" term containing $g$ and depending only on the point values $u_{\alpha}$ is of lower order with respect to strong interactions but of the same order as the weak interactions.

We suppose that there are $N$ infinite connected components of the graph of points linked by strong interactions, which we denote by $C_{1}, \ldots, C_{N}$. Note that weak interactions in $\mathcal{N}_{0}$ are due either to the existence of "weak sites" or to weak bonds between different "strong components" and, if we have more than one strong graph, the interactions in $\mathcal{N}_{0}$ are present also in the absence of a weak component. We will describe the asymptotic behavior of energies (1) using the notation and techniques of $\Gamma$-convergence (see, e.g., [Braides 2002; 2006]).

If we consider only the strong interactions restricted to each strong connected component $C_{j}$, we obtain energies

$$
F_{\varepsilon}^{j}(u)=\sum_{(\alpha, \beta) \in \mathcal{E} \mathcal{N}_{1}^{j} \cap(\Omega \times \Omega)} \varepsilon^{d-1} a_{\alpha \beta}^{\varepsilon}\left(u_{\alpha}-u_{\beta}\right)^{2},
$$

where $\mathcal{N}_{1}^{j}$ is the restriction to $C_{j} \times C_{j}$ of the set $\mathcal{N}_{1}$. This is a discrete analog of an energy on a perforated domain, the perforation being $\mathbb{Z}^{d} \backslash C_{j}$.

We prove an extension lemma that allows us to define for each $j \in\{1, \ldots, N\}$ a discrete-to-continuum convergence of (the restriction to $C_{j}$ of) a sequence of functions $u^{\varepsilon}$ to a function $u^{j} \in \mathrm{BV}(\Omega ;\{ \pm 1\})$, which is compact under an equiboundedness assumption for the energies $F_{\varepsilon}^{j}\left(u^{\varepsilon}\right)$. Thanks to this lemma, such energies behave as ferromagnetic energies with positive coefficients on the whole of $\mathbb{Z}^{d}$, which can be homogenized thanks to [Braides and Piatnitski 2013]; i.e., their $\Gamma$-limit with respect to the convergence $u^{\varepsilon} \rightarrow u^{j}$ exists and is of the form

$$
F^{j}\left(u^{j}\right)=\int_{S\left(u^{j}\right) \cap \Omega} f_{\text {hom }}^{j}\left(v_{u^{j}}\right) d \mathscr{H}^{d-1}
$$


where $S\left(u^{j}\right)$ is the set of jump points of $u^{j}$, which can also be interpreted as the interface between $\left\{u^{j}=1\right\}$ and $\left\{u^{j}=-1\right\}$.

Taking into account separately the restrictions of $u^{\varepsilon}$ to all of the components $C_{j}$, we define a vector-valued limit function $u=\left(u^{1}, \ldots, u^{N}\right)$ and a convergence $u^{\varepsilon} \rightarrow u$ and consider the $\Gamma$-limit of the whole energy with respect to that convergence. The combination of the weak interactions and the forcing term gives rise to a term of the form

$$
\int_{\Omega} \varphi(u) d x
$$

depending on the values of all components of $u$. In the case that $\bigcup_{j=1}^{N} C_{j}$ is all of $\mathbb{Z}^{d}$, the function $\varphi\left(z^{1}, \ldots, z^{N}\right)$ is simply computed as the average of the $T$-periodic function

$$
i \mapsto \sum_{k \in \mathbb{Z}^{d}} a_{i k}\left(u_{i}-u_{k}\right)^{2}+g\left(u_{i}\right)
$$

where $u$ takes the value $z^{j}$ on $C_{j}$. Note that with this condition only (weak) interactions between different $C_{j}$ are taken into account. Note moreover that the restriction of the last term $g$ to $\varepsilon C_{j}$ is continuously converging to

$$
K_{j} \int_{\Omega} g\left(u^{j}\right) d x
$$

where $K_{j}=T^{-d} \#\left\{i \in C^{j}: i \in\{0, \ldots, T\}^{d}\right\}$ is the percentage of sites in $C_{j}$. In general, $\varphi$ is obtained by optimizing the combined effect of weak pair interactions and $g$ on the free sites in the complement of all $C_{j}$.

Such different interactions can be summed up to describe the $\Gamma$-limit of $F_{\varepsilon}$ that finally takes the form

$$
F_{\mathrm{hom}}(u)=\int_{S(u) \cap \Omega} f_{\mathrm{hom}}\left(u^{+}, u^{-}, v_{u}\right) d \mathscr{H}^{d-1}+\int_{\Omega} \varphi(u) d x,
$$

where $f_{\text {hom }}\left(u^{+}, u^{-}, v\right)=\frac{1}{2} \sum_{j=1}^{N} f_{\text {hom }}^{j}(v)\left|u_{j}^{+}-u_{j}^{-}\right|$.

We note that the presence of two terms of different dimensions in the limit highlights the combination of bulk homogenization effects due to periodic oscillations besides the optimization of the interfacial structure. The effect of those oscillations on the variational motions of such systems (in the sense of [Ambrosio et al. 2008; Braides 2014b]) is addressed in [Braides and Solci 2015]. With respect to [Braides et al. 2015], we remark that the case of spin systems allows a very easy proof of an extension lemma from connected discrete sets and at the same time permits us to highlight the possibility to include a weak phase with antiferromagnetic interactions, optimized by microscopic oscillations. 
Discrete problems modeling high-contrast media in the case of elastic energies have recently been considered in [Braides et al. 2015], but double-porosity homogenization in a continuum framework is a long-standing issue. The interest in doubleporosity systems came at first from geophysics. The notion of double porosity, or double permeability, is borne from studies carried out on naturally fractured porous rocks such as oil fields. The benefits of describing oil flow and stock capacity in these kinds of soils justified theoretical studies undertaken during the 1960s. The double-porosity model was first introduced by [Barenblatt et al. 1960], and it has been used since in a wide range of engineering specialties. The first rigorous mathematical result on the subject was obtained in [Arbogast et al. 1990], where a linear parabolic equation with asymptotically degenerating coefficients was considered. This result was subsequently generalized in [Panasenko 1991; Bourgeat et al. 1996; 1998; 1999; Sandrakov 1999a; 1999b; Pankratov and Piatnitski 2002; Marchenko and Khruslov 2006] also for nonperiodic domains and various rates of contrast. On the physical level of rigor, double-porosity models were studied in [Panfilov 2000]. Linear double-porosity models with thin fissures were considered in [Pankratov and Rybalko 2003; Amaziane et al. 2009b]. The singular doubleporosity model was considered in [Bourgeat et al. 2003]. The works [Bourgeat et al. 1999; Marchenko and Khruslov 2006; Pankratov and Rybalko 2003; Amaziane et al. 2009b] are carried out in the framework of Khruslov's mesoscopic energy characteristic methods. In addition, note that the double-porosity model was also obtained using the two-scale convergence method in [Hornung 1997]. Elliptic and parabolic nonlinear double-porosity models, including homogenization in variable Sobolev spaces, were also obtained in [Pankratov et al. 2003; Amaziane et al. 2006; 2009a; Choquet and Pankratov 2010]. Finally, the double-porosity models of multiphase flows, including the nonequilibrium ones, were also obtained in [Choquet 2004; Yeh 2006; Amaziane and Pankratov 2016; Konyukhov and Pankratov 2015] (see also [Hornung 1997] and the references therein). A reformulation in terms of $\Gamma$-convergence can be found in [Braides et al. 2004] with related results for nonconvex integrands. An approach using $\Gamma$-convergence and a two-scale formulation at the same time is given in [Cherdantsev and Cherednichenko 2012]. Double-porosity models for interfacial energies on the continuum were previously examined in [Solci 2009; 2012; Braides and Solci 2013].

The results in the present paper may be regarded as a geometrically simplified model of continuum ones (but with more freedom in the lattice interactions), but the same framework may also be useful for other discrete models actually developed in mechanics. Among them are pantographic systems made of beams and used for modeling of some metamaterials [Seppecher et al. 2011] and investigations of two- and three-dimensional lattices in order to develop models used in nano- and micromechanics. 
The plan of the paper is the following. In Section 2, we introduce the geometric setting, identifying the "strong" and possibly "weak" phases of the lattice network, and define the microscopic energy. In Section 3, we prove a compactness theorem and a homogenization result for each separate strong phase. The resulting energies will provide the interfacial energy part of the limit. In Section 4, we define the interaction term between the strong phases by proving an asymptotic formula. The main convergence result is stated and proved in Section 5, where the compactness theorem in Section 3 applied to each strong phase is used to define a multiphase limit. Finally in Section 6, some simple examples are provided, which in particular also exhibit nontrivial limits in dimensions 1 and 2.

\section{Notation}

The numbers $d, m, T$, and $N$ are positive integers. We introduce a $T$-periodic label function $J: \mathbb{Z}^{d} \rightarrow\{0,1, \ldots, N\}$ and the corresponding sets of sites

$$
A_{j}=\left\{k \in \mathbb{Z}^{d}: J(k)=j\right\}, \quad j=0, \ldots, N .
$$

Sites interact through possibly long- (but finite-)range interactions, whose range is defined through a system $P^{j}=\left\{P_{k}^{j}\right\}$ of finite subsets $P_{k}^{j} \subset \mathbb{Z}^{d}$ for $j=0, \ldots, N$ and $k \in A_{j}$. We suppose

- (T-periodicity) $P_{k+m}^{j}=P_{k}^{j}$ for all $m \in T \mathbb{Z}^{d}$ and

- (symmetry) if $k \in A_{j}$ for $j=1, \ldots, N$ (hard components) and $i \in P_{k}^{j}$, then $k+i \in A_{j}$ and $-i \in P_{k+i}^{j}$, and $0 \in P_{k}^{j}$.

We say that two points $k, k^{\prime} \in A_{j}$ are $P^{j}$-connected in $A_{j}$ if there exists a path $\left\{k_{n}\right\}_{n=0, \ldots, K}$ such that $k_{n} \in A_{j}, k_{0}=k, k_{K}=k^{\prime}$, and $k_{n}-k_{n-1} \in P_{k_{n-1}}^{j}$.

We suppose

- (connectedness) there exists a unique infinite $P^{j}$-connected component of each $A_{j}$ for $j=1, \ldots, N$, which we denote by $C_{j}$.

Clearly, the connectedness assumption is not a modeling restriction upon introducing more labeling parameters if the number of infinite connected components is finite. Note that we do not make any assumptions on $A_{0}$ and $P^{0}$. In particular, if $k \in A_{j}$ for $j=0, \ldots, N$ and $i \in P_{k}^{0}$, then $k+i$ may belong to any $A_{j^{\prime}}$ with $j^{\prime} \neq j$.

We consider the following sets of bonds between sites in $\mathbb{Z}^{d}$ : for $j=1, \ldots, N$

$$
N_{j}=\left\{\left(k, k^{\prime}\right): k, k^{\prime} \in A_{j}, k^{\prime}-k \in P_{k}^{j} \backslash\{0\}\right\}
$$

and for $j=0$

$$
N_{0}=\left\{\left(k, k^{\prime}\right): k^{\prime}-k \in P_{k}^{0} \backslash\{0\}, J(k) J\left(k^{\prime}\right)=0 \text { or } J(k) \neq J\left(k^{\prime}\right)\right\} .
$$


Note that the set $N_{0}$ takes into account interactions not only among points of the set $A_{0}$ but also among pairs of points in different $A_{j}$. More refined notation could be introduced by defining a range of interactions $P^{i j}$ and the corresponding sets $N_{i j}$, in which case the sets $N_{j}$ would correspond to $N_{j j}$ for $j=1, \ldots, N$ and $N_{0}$ to the union of the remaining sets. However, for simplicity of presentation, we limit our notation to a single index.

We consider interaction energy densities associated with positive numbers $a_{k k^{\prime}}$ for $k, k^{\prime} \in \mathbb{Z}^{d}$ and the forcing term $g$. We suppose that for all $k, k^{\prime} \in \mathbb{Z}^{d}$

- (coerciveness on the hard phase) there exists $c>0$ such that $a_{k k^{\prime}} \geq c>0$ if $k \in C_{j}$ and $k^{\prime}-k \in P_{k}^{j}$ for $j \geq 1$,

- (T-periodicity) $a_{k+m k^{\prime}+m}=a_{k k^{\prime}}$ for all $m \in T \mathbb{Z}^{d}$,

- (symmetry) $a_{k^{\prime} k}=a_{k k^{\prime}}$, and

- $(T$-periodicity of the forcing term) $g(k+m, 1)=g(k, 1)$ and $g(k+m,-1)=$ $g(k,-1)$ for all $m \in T \mathbb{Z}^{d}$.

Note that we do not suppose that the $a_{k k^{\prime}}$ are positive for weak interactions. They can be negative as well, thus favoring oscillations in the weak phase.

Given $\Omega$, a bounded regular open subset of $\mathbb{R}^{d}$, for $u:(1 / \varepsilon) \Omega \cap \mathbb{Z}^{d} \rightarrow\{+1,-1\}$, we define the energies

$$
\begin{aligned}
F_{\varepsilon}(u)=F_{\varepsilon}\left(u, \frac{1}{\varepsilon} \Omega\right)= & \sum_{j=1}^{N} \sum_{\left(k, k^{\prime}\right) \in \mathcal{N}_{j}^{\varepsilon}(\Omega)} \varepsilon^{d-1} a_{k k^{\prime}}\left(u_{k}-u_{k^{\prime}}\right)^{2} \\
& +\sum_{\left(k, k^{\prime}\right) \in \mathcal{N}_{0}^{\varepsilon}(\Omega)} \varepsilon^{d} a_{k k^{\prime}}\left(u_{k}-u_{k^{\prime}}\right)^{2}+\sum_{k \in Z^{\varepsilon}(\Omega)} \varepsilon^{d} g\left(k, u_{k}\right),
\end{aligned}
$$

where

$$
\mathcal{N}_{j}^{\varepsilon}(\Omega)=N_{j} \cap \frac{1}{\varepsilon}(\Omega \times \Omega), \quad j=0, \ldots, N, \quad Z^{\varepsilon}(\Omega)=\mathbb{Z}^{d} \cap \frac{1}{\varepsilon} \Omega .
$$

The first sum in the energy takes into account all interactions between points in $A_{j}$ (hard phases), which are supposed to scale differently than those between points in $A_{0}$ (soft phase) or between points in different phases. The latter are contained in the second sum. The third sum is a zero-order term taking into account all types of phases with the same scaling.

Note that the first sum may also take into account points in $A_{j} \backslash C_{j}$, which form "islands" of the hard phase $P^{j}$-disconnected from the corresponding infinite component. Furthermore, in this energy, we may have sites that do not interact at all with hard phases. 
Remark 2.1 (choice of the parameter space). The energy is defined on discrete functions parametrized on $(1 / \varepsilon) \Omega \cap \mathbb{Z}^{d}$. The choice of this notation, rather than interpreting $u$ as defined on $\Omega \cap \varepsilon \mathbb{Z}^{d}$, allows a much easier notation for the coefficients, which in this way are $\varepsilon$-independent rather than obtained by scaling as in (2).

\section{Homogenization of perforated discrete domains}

In this section, we separately consider the interactions in each infinite connected component of the hard phases introduced above. To that end, we fix one of the indices $j$, with $j>0$, dropping it in the notation of this section (in particular, we use the symbol $C$ in place of $C_{j}$, etc.), and define the energies

$$
\mathscr{F}_{\varepsilon}(u)=\mathscr{F}_{\varepsilon}\left(u, \frac{1}{\varepsilon} \Omega\right)=\sum_{\left(k, k^{\prime}\right) \in N_{C}^{\varepsilon}(\Omega)} \varepsilon^{d-1} a_{k k^{\prime}}\left(u_{k}-u_{k^{\prime}}\right)^{2},
$$

where

$$
N_{C}^{\varepsilon}(\Omega)=\left\{\left(k, k^{\prime}\right) \in(C \times C) \cap \frac{1}{\varepsilon}(\Omega \times \Omega): k^{\prime}-k \in P_{k}, k \neq k^{\prime}\right\} .
$$

We also introduce the notation $C^{\varepsilon}(\Omega)=C \cap(1 / \varepsilon) \Omega$.

Definition 3.1. We define the piecewise-constant interpolation of a function $u$ : $\mathbb{Z}^{d} \cap(1 / \varepsilon) \Omega \rightarrow \mathbb{R}^{m}, k \mapsto u_{k}$, as

$$
u(x)=u_{\lfloor x / \varepsilon\rfloor},
$$

where $\lfloor y\rfloor=\left(\left\lfloor y_{1}\right\rfloor, \ldots,\left\lfloor y_{d}\right\rfloor\right)$ and $\lfloor s\rfloor$ stands for the integer part of $s$. The convergence of a sequence $\left(u^{\varepsilon}\right)$ of discrete functions is understood as the $L_{\text {loc }}^{1}(\Omega)$ convergence of these piecewise-constant interpolations. Note that, since we consider local convergence in $\Omega$, the value of $u(x)$ close to the boundary in not involved in the convergence process.

We prove an extension and compactness lemma with respect to the convergence of piecewise-constant interpolations.

Lemma 3.2 (extension and compactness). Let $C$ be a T-periodic subset of $\mathbb{Z}^{d}$ $P$-connected in the notation of the previous section, and let $u^{\varepsilon}: \mathbb{Z}^{d} \cap(1 / \varepsilon) \Omega \rightarrow$ $\{+1,-1\}$ be a sequence such that

$$
\sup _{\varepsilon} \varepsilon^{d-1} \#\left\{\left(k, k^{\prime}\right) \in N_{C}^{\varepsilon}(\Omega): u_{k}^{\varepsilon} \neq u_{k^{\prime}}^{\varepsilon}\right\}<+\infty .
$$

Then there exists a sequence $\tilde{u}^{\varepsilon}: \mathbb{Z}^{d} \cap(1 / \varepsilon) \Omega \rightarrow \mathbb{R}^{m}$ such that $\tilde{u}_{k}^{\varepsilon}=u_{k}^{\varepsilon}$ if $k \in C^{\varepsilon}(\Omega)$ and $\operatorname{dist}(k, \partial(1 / \varepsilon) \Omega)>c=c(P)$ with $\tilde{u}^{\varepsilon}$ converging to some $u \in \mathrm{BV}_{\text {loc }}(\Omega ;\{+1,-1\})$ up to subsequences.

Proof. For a fixed $M \in \mathbb{N}$ and $j \in \mathbb{Z}^{d}$, we consider the discrete cubes of side length $M$

$$
Q_{M}(j):=j M+\{0, M-1\}^{d} .
$$


For each $j$, we also define the cube

$$
Q_{3 M}^{\prime}(j)=\bigcup_{\|i-j\|_{\infty} \leq 1} Q_{M}(i),
$$

which is a discrete cube centered at $Q_{M}(j)$ and with side length $3 M$.

For all $\varepsilon$, we consider the family

$$
2_{M}^{\varepsilon}:=\left\{Q_{M}(j): j \in \mathbb{Z}^{d}, Q_{3 M}^{\prime}(j) \subset \frac{1}{\varepsilon} \Omega\right\} .
$$

We suppose that $M$ is large enough such that, if $k, k^{\prime} \in Q_{M}(j) \cap C$, then there exists a $P$-path connecting $k$ and $k^{\prime}$ contained in $Q_{3 M}^{\prime}(j)$. The existence of such $M$ follows easily from the connectedness hypotheses. Indeed, we may take $M$ as the length of the longest shortest $P$-path connecting two points in $C$ with distance not greater than $2 \sqrt{d}$ (in particular belonging to neighboring periodicity cubes) and construct such a $P$-path by concatenating a family of those shortest paths.

We define the set of indices

$$
\mathscr{Y}_{\varepsilon}=\left\{j \in \mathbb{Z}^{d}: Q_{M}(j) \in \mathscr{2}_{M}^{\varepsilon} \text { and } u^{\varepsilon} \text { is not constant on } C \cap Q_{M}(j)\right\} .
$$

By our choice of $M$, if $j \in \mathscr{Y}_{\varepsilon}$, then there exist $k, k^{\prime} \in Q_{3 M}^{\prime}(j) \cap C$ with $k^{\prime}-k \in P$ such that $u_{k}^{\varepsilon} \neq u_{k^{\prime}}^{\varepsilon}$. Let

$$
K:=\sup _{\varepsilon} \varepsilon^{d-1} \#\left\{\left(k, k^{\prime}\right) \in N_{C}^{\varepsilon}(\Omega): u_{k}^{\varepsilon} \neq u_{k^{\prime}}^{\varepsilon}\right\} .
$$

Then we deduce that

$$
\# \mathscr{S}_{\varepsilon} \leq 3^{d} K \frac{1}{\varepsilon^{d-1}}
$$

(the factor $3^{d}$ comes from the fact that $k, k^{\prime} \in Q_{3 M}^{\prime}(j)$ for $3^{d}$ possible $j$ ).

We define $\tilde{u}^{\varepsilon}=\left\{\begin{array}{cl}\text { constant value of } u^{\varepsilon} \text { on } Q_{M}(j) \cap C & \text { on } Q_{M}(j) \text { if } Q_{M}(j) \in \mathscr{2}_{M}^{\varepsilon} \text { and } j \notin \mathscr{Y}_{\varepsilon}, \\ u^{\varepsilon} & \text { elsewhere. }\end{array}\right.$

This will be the required extension. However, we will prove the convergence of $\tilde{u}^{\varepsilon}$ as a consequence of the convergence of the functions

$$
v^{\varepsilon}= \begin{cases}\tilde{u}^{\varepsilon} & \text { on } Q_{M}(j) \text { if } Q_{M}(j) \in \mathscr{2}_{M}^{\varepsilon} \text { and } j \notin \mathscr{Y}_{\varepsilon}, \\ 1 & \text { elsewhere. }\end{cases}
$$

By (11), we have that for fixed $\Omega^{\prime} \Subset \Omega$

$$
\left\|v^{\varepsilon}-\tilde{u}^{\varepsilon}\right\|_{L^{1}\left(\Omega^{\prime}\right)}=O(\varepsilon)
$$

(recall that we identify the function with its scaled interpolations in $L^{1}(\Omega)$ ).

If the value of $v^{\varepsilon}$ differs on two neighboring $Q_{M}(j)$ and $Q_{M}\left(j^{\prime}\right)$ with $\left\|j-j^{\prime}\right\|_{1}=$ 1 , then upon taking a suitable larger $M$, we may also suppose that there exist 
$k, k^{\prime} \in\left(Q_{3 M}^{\prime}(j) \cup Q_{3 M}^{\prime}(j)\right) \cap C$ with $k-k^{\prime} \in P$ and $u_{k}^{\varepsilon} \neq u_{k^{\prime}}^{\varepsilon}$. Arguing as for (11), we deduce that the number of such $j$ is $O\left(\varepsilon^{1-d}\right)$ so that

$$
\mathscr{H}^{d-1}\left(\partial\left\{v^{\varepsilon}=1\right\} \cap \Omega^{\prime}\right)=O(1),
$$

which implies the compactness of the family $\left(v^{\varepsilon}\right)$ in $\mathrm{BV}_{\text {loc }}(\Omega)$.

The compactness theorem above proves that the domain of the limit is functions $u \in \mathrm{BV}(\Omega,\{+1,-1\})$, which can be identified with the sets of finite perimeter $E=\{u=1\}$. In this case, the set of discontinuity points $S(u)$ coincides, up to sets of $\mathscr{H}^{n-1}$-measure 0 , with the reduced boundary $\partial^{*}\{u=1\}$, whose inner normal we denote by $v$ [Braides 1998].

Theorem 3.3 (homogenization on discrete perforated domains). The energies $\mathscr{F}_{\varepsilon}$ defined in (8) $\Gamma$-converge with respect to the $L_{\text {loc }}^{1}(\Omega)$ topology to the energy

$$
\mathscr{F}_{\text {hom }}(u)=\int_{\Omega \cap \partial^{*}\{u=1\}} f_{\text {hom }}(v) d \mathscr{H}^{d-1},
$$

defined on $u \in \mathrm{BV}(\Omega,\{+1,-1\})$, where the energy density $f_{\text {hom }}$ satisfies

$$
\begin{aligned}
& f_{\mathrm{hom}}(v)= \\
& \lim _{T \rightarrow+\infty} \frac{1}{T^{d-1}} \inf \left\{\sum_{\left(k, k^{\prime}\right) \in \tilde{N}_{C}\left(Q_{T}^{v}\right)} a_{k k^{\prime}}\left(u_{k}-u_{k^{\prime}}\right)^{2}: u_{k}=\operatorname{sign}\langle k, v\rangle \text { if } k \notin Q_{T}^{v}\right\},
\end{aligned}
$$

where

$$
\operatorname{sign} x=\left\{\begin{aligned}
1 & \text { if } x>0 \\
-1 & \text { if } x \leq 0
\end{aligned}\right.
$$

$Q^{v}$ is a cube centered at 0 and with one side orthogonal to $v, Q_{T}^{v}=T Q^{v}$, and $\widetilde{N}_{C}\left(Q_{T}^{v}\right)$ denotes all pairs in $\left(k, k^{\prime}\right) \in N_{C}^{1}\left(\mathbb{R}^{d}\right)$ such that either $k \in Q_{T}^{v}$ or $k^{\prime} \in Q_{T}^{v}$.

Proof. In [Braides and Piatnitski 2013], this theorem is proved under the additional assumption that the energies $\mathscr{F}_{\varepsilon}$ are equicoercive with respect to the weak BVconvergence. This assumption can be substituted with Lemma 3.2. Indeed, if $u^{\varepsilon}$ is a sequence converging to $u$ in $L_{\text {loc }}^{1}(\Omega)$ and with equibounded energies, then by Lemma 3.2, we may find a sequence $\tilde{u}^{\varepsilon}$ coinciding with $u^{\varepsilon}$ on $C^{\varepsilon}\left(\Omega^{\prime}\right)$ for every fixed $\Omega^{\prime} \Subset \Omega$ and $\varepsilon$ sufficiently small and converging to some $\tilde{u}$ in $\operatorname{BV}(\Omega ;\{ \pm 1\})$. Since $\tilde{u}^{\varepsilon}=u_{\varepsilon}$ on $C^{\varepsilon}\left(\Omega^{\prime}\right)$, we have that $\tilde{u}=u$ and $\mathscr{F}_{\varepsilon}\left(\tilde{u}^{\varepsilon},(1 / \varepsilon) \Omega^{\prime}\right)=\mathscr{F}_{\varepsilon}\left(u^{\varepsilon},(1 / \varepsilon) \Omega^{\prime}\right)$. Then we can give a lower estimate on each $\Omega^{\prime}$ fixed using the proof of [Braides and Piatnitski 2013] and hence on $\Omega$ by internal approximation. Note that neither the proof of the existence of the limit in (13) therein nor the construction of the recovery sequences depends on the coerciveness assumption, so the proof is complete. 


\section{Definition of the interaction term}

The homogenization result in Theorem 3.3 will describe the contribution of the hard phases to the limiting behavior of energies $F_{\varepsilon}$. We now characterize their interactions with the soft phase.

For all positive integers $M$ and $z_{1}, \ldots, z_{N} \in\{+1,-1\}$, we define the minimum problem

$\varphi_{M}\left(z_{1}, \ldots, z_{N}\right)=\frac{1}{M^{d}} \min \left\{\sum_{\left(k, k^{\prime}\right) \in N_{0}\left(Q_{M}\right)} a_{k k^{\prime}}\left(v_{k}-v_{k^{\prime}}\right)^{2}+\sum_{k \in Z\left(Q_{M}\right)} g\left(k, v_{k}\right): v \in \mathscr{V}_{M}\right\}$,

where

$$
Q_{M}=\left[-\frac{M}{2}, \frac{M}{2}\right)^{d}, \quad N_{0}\left(Q_{M}\right)=N_{0} \cap\left(Q_{M} \times Q_{M}\right), \quad Z\left(Q_{M}\right)=\mathbb{Z}^{d} \cap Q_{M}
$$

and the minimum is taken over the set $\mathscr{V}_{M}=\mathscr{V}_{M}\left(z_{1}, \ldots, z_{N}\right)$ of all $v$ constant on each connected component of $A_{j} \cap Q_{M}$ and $v=z_{j}$ on $C_{j}$ for $j=1, \ldots N$.

Proposition 4.1. The limit $\varphi$ of $\varphi_{M}$ as $M \rightarrow+\infty$ exists.

Proof. We first show that

$$
\varphi_{K M} \geq \varphi_{M} \quad \text { for all } K \in \mathbb{N} .
$$

To that end, let $\bar{v}$ be a minimizer for $\varphi_{K M}\left(z_{1}, \ldots, z_{N}\right)$. Then we have $K^{d} M^{d} \varphi_{K M}\left(z_{1}, \ldots, z_{N}\right)$

$$
\begin{aligned}
= & \sum_{\left(k, k^{\prime}\right) \in N_{0}\left(Q_{K M}\right)} a_{k k^{\prime}}\left(\bar{v}_{k}-\bar{v}_{k^{\prime}}\right)^{2}+\sum_{k \in Z\left(Q_{K M}\right)} g\left(k, \bar{v}_{k}\right) \\
= & \sum_{l \in \mathbb{Z}^{d} \cap Q_{K}}\left(\sum_{\left(k, k^{\prime}\right) \in N_{0}\left(Q_{M}+l M\right)} a_{k k^{\prime}}\left(\bar{v}_{k}-\bar{v}_{k^{\prime}}\right)^{2}+\sum_{k \in Z\left(Q_{M}+l M\right)} g\left(k, \bar{v}_{k}\right)\right) \\
& +\sum_{\left(k, k^{\prime}\right) \in N_{0}\left(Q_{K M}\right) \backslash \bigcup_{l} N_{0}\left(Q_{M}+l M\right)} a_{k k^{\prime}}\left(\bar{v}_{k}-\bar{v}_{k^{\prime}}\right)^{2} \\
\geq & \sum_{l \in \mathbb{Z}^{d} \cap Q_{K}}\left(\sum_{\left(k, k^{\prime}\right) \in N_{0}\left(Q_{M}+l M\right)} a_{k k^{\prime}}\left(\bar{v}_{k}-\bar{v}_{k^{\prime}}\right)^{2}+\sum_{k \in Z\left(Q_{M}+l M\right)} g\left(k, \bar{v}_{k}\right)\right) .
\end{aligned}
$$

Let $\bar{l} \in \mathbb{Z}^{d} \cap Q_{K}$ minimize the expression in parentheses. Then we deduce

$$
\begin{aligned}
K^{d} M^{d} \varphi_{K M}\left(z_{1}, \ldots, z_{N}\right) & \geq K^{d}\left(\sum_{\left(k, k^{\prime}\right) \in N_{0}\left(Q_{M}+\bar{l} M\right)} a_{k k^{\prime}}\left(\bar{v}_{k}-\bar{v}_{k^{\prime}}\right)^{2}+\sum_{k \in Z\left(Q_{M}+\bar{l} M\right)} g\left(k, \bar{v}_{k}\right)\right),
\end{aligned}
$$

from which (16) follows by taking $v_{k}=\bar{v}_{k-\bar{l} M}$ in the computation of $\varphi_{M}\left(z_{1}, \ldots, z_{N}\right)$. 
We remark that for $L \geq L^{\prime}$ we have

$$
L^{d} \varphi_{L} \geq\left(L^{\prime}\right)^{d} \varphi_{L^{\prime}}-\max |g|\left(L^{d}-\left(L^{\prime}\right)^{d}\right) .
$$

Hence, fixing $n, L$, and $M$ with $L \geq M 2^{n}$ and taking $L^{\prime}=\left\lfloor L /\left(M 2^{n}\right)\right\rfloor M 2^{n}$ in (17), we have, using (16) with $K=\left\lfloor L /\left(M 2^{n}\right)\right\rfloor 2^{n}$

$$
\begin{aligned}
\varphi_{L} & \geq \frac{1}{L^{d}}\left(\left\lfloor\frac{L}{M 2^{n}}\right\rfloor M 2^{n}\right)^{d} \varphi_{\left\lfloor L /\left(M 2^{n}\right)\right\rfloor M 2^{n}}-\max |g|\left(1-\left(\left\lfloor\frac{L}{M 2^{n}}\right\rfloor \frac{M 2^{n}}{L}\right)^{d}\right) \\
& \geq\left(\left\lfloor\frac{L}{M 2^{n}}\right\rfloor \frac{M 2^{n}}{L}\right)^{d} \varphi_{M}-\max |g|\left(1-\left(\left\lfloor\frac{L}{M 2^{n}}\right\rfloor \frac{M 2^{n}}{L}\right)^{d}\right) .
\end{aligned}
$$

Letting $L \rightarrow+\infty$, we then obtain

$$
\liminf _{L \rightarrow+\infty} \varphi_{L} \geq \varphi_{M}
$$

and the conclusion follows by taking the upper limit in $M$.

Let $R$ be defined by

$$
R=\max \left\{\left|k-k^{\prime}\right|: k, k^{\prime} \in A_{j} \backslash C_{j} \text { that are } P^{j} \text {-connected, } j=1, \ldots, N\right\},
$$

and for all $M$ positive integer, set

$$
D_{M}=\bigcup_{j=1}^{N} \bigcup\left\{P^{j} \text {-connected components } B \text { of } A_{j} \backslash C_{j} \text { not intersecting } Q_{M-R}\right\} \text {. }
$$

For all $z_{1}, \ldots, z_{N} \in\{+1,-1\}$, we define

$$
\begin{array}{r}
\tilde{\varphi}_{M}\left(z_{1}, \ldots, z_{N}\right)=\frac{1}{M^{d}} \min \left\{\sum_{\left(k, k^{\prime}\right) \in N_{0}\left(Q_{M}\right)} a_{k k^{\prime}}\left(v_{k}-v_{k^{\prime}}\right)^{2}+\sum_{k \in Z\left(Q_{M}\right)} g\left(k, v_{k}\right)\right. \\
\left.: v \in \mathscr{V}_{M}, v_{k}=1 \text { if } k \in D_{M}\right\} .
\end{array}
$$

Proposition 4.2. There is a positive constant $c$ independent of $M$ such that

$$
\widetilde{\varphi}_{M} \geq \varphi_{M} \geq \widetilde{\varphi}_{M}-\frac{c}{M} .
$$

Proof. The first inequality is trivial. To prove the second, let $\bar{v}$ be a minimizer for $\varphi_{M}\left(z_{1}, \ldots, z_{N}\right)$ and define $v$ by

$$
v_{k}= \begin{cases}1 & \text { if } k \in D_{M} \\ \bar{v}_{k} & \text { otherwise }\end{cases}
$$


Using $v$ as a test function for $\widetilde{\varphi}_{M}\left(z_{1}, \ldots, z_{N}\right)$, we obtain

$$
\begin{aligned}
M^{d} \widetilde{\varphi}_{M}\left(z_{1}, \ldots, z_{N}\right) \leq & \sum_{\left(k, k^{\prime}\right) \in N_{0}\left(Q_{M}\right), k, k^{\prime} \notin D_{M}} a_{k k^{\prime}}\left(v_{k}-v_{k^{\prime}}\right)^{2}+\sum_{k \in Z\left(Q_{M}\right) \backslash D_{M}} g\left(k, v_{k}\right) \\
& +2 \sum_{\left(k, k^{\prime}\right) \in N_{0}\left(Q_{M}\right), k \in D_{M}} a_{k k^{\prime}}\left(v_{k}-v_{k^{\prime}}\right)^{2}+\sum_{k \in Z\left(Q_{M}\right) \cap D_{M}} g\left(k, v_{k}\right) \\
\leq & \sum_{\left(k, k^{\prime}\right) \in N_{0}\left(Q_{M}\right), k, k^{\prime} \notin D_{M}} a_{k k^{\prime}}\left(\bar{v}_{k}-\bar{v}_{k^{\prime}}\right)^{2}+\sum_{k \in Z\left(Q_{M}\right) \backslash D_{M}} g\left(k, \bar{v}_{k}\right) \\
& +\sum_{\left(k, k^{\prime}\right) \in N_{0}\left(Q_{M}\right), k \in D_{M}} a_{k k^{\prime}}+\sum_{k \in Z\left(Q_{M}\right) \cap D_{M}} g(k, 1) \\
\leq & M^{d} \varphi_{M}\left(z_{1}, \ldots, z_{N}\right)+\# D_{M} \# P_{0} \max a_{i j}+\# D_{M} 2 \max |g| .
\end{aligned}
$$

As \# $D_{M} \leq 2^{d} M^{d-1} R$, the result follows with $c=2^{d} R\left(\# P_{0} \max a_{i j}+2 \max |g|\right)$.

\section{Statement of the convergence result}

We now have all the ingredients to characterize the asymptotic behavior of $F_{\varepsilon}$ defined in (6).

Definition 5.1 (multiphase discrete-to-continuum convergence). We define the convergence

$$
u^{\varepsilon} \rightarrow\left(u^{1}, \ldots, u^{N}\right)
$$

as the $L_{\text {loc }}^{1}\left(\Omega ; \mathbb{R}^{m}\right)$ convergence $\tilde{u}_{j}^{\varepsilon} \rightarrow u^{j}$ of the extensions of the restrictions of $u^{\varepsilon}$ to $C_{j}$ as in Lemma 3.2, which is a compact convergence as ensured by that lemma.

The total contribution of the hard phases will be given separately by the contribution on the infinite connected components and the finite ones. The first one is obtained by independently computing the limit relative to the energy restricted to each component

$$
\mathscr{F}_{\varepsilon}^{j}(u)=\sum_{\left(k, k^{\prime}\right) \in N_{j}^{\varepsilon}(\Omega)} \varepsilon^{d-1} a_{k k^{\prime}}\left(v_{k}-v_{k^{\prime}}\right)^{2}
$$

where

$$
N_{j}^{\varepsilon}(\Omega)=N_{C_{j}}^{\varepsilon}(\Omega)=\left\{\left(k, k^{\prime}\right) \in\left(C_{j} \times C_{j}\right) \cap \frac{1}{\varepsilon}(\Omega \times \Omega): k-k^{\prime} \in P_{k}^{j}, k \neq k^{\prime}\right\},
$$

which is characterized by Theorem 3.3 as

$$
\mathscr{F}_{\text {hom }}^{j}(u)=\int_{\Omega \cap \partial^{*}\{u=1\}} f_{\text {hom }}^{j}(v) d \mathscr{H}^{d-1} .
$$

In Section 4, we introduced the energy density $\varphi$, which describes the interactions between the hard phases. Taking all contributions into account, we may state the following convergence result. 
Theorem 5.2 (double-porosity homogenization). Let $\Omega$ be a Lipschitz bounded open set, and let $F_{\varepsilon}$ be defined by (6) with the notation of Section 2. Then the $\Gamma$-limit of $F_{\varepsilon}$ with respect to the convergence (21) exists, and it equals

$$
F_{\mathrm{hom}}\left(u^{1}, \ldots, u^{N}\right)=\sum_{j=1}^{N} \int_{\Omega \cap \partial^{*}\left\{u^{j}=1\right\}} f_{\mathrm{hom}}^{j}(v) d \mathscr{H}^{d-1}+\int_{\Omega} \varphi\left(u^{1}, \ldots, u^{N}\right) d x
$$

on functions $u=\left(u^{1}, \ldots, u^{N}\right) \in(\mathrm{BV}(\Omega ;\{1,-1\}))^{N}$, where $\varphi$ is defined in Proposition 4.1 and $f_{\text {hom }}^{j}$ are defined by (24).

Note that there is no contribution of the finite connected components of $A_{j}$.

Remark 5.3 (nonhomogeneous lower-order term). In our hypotheses, the lowerorder term $g$ depends on the fast variable $k$, which is integrated out in the limit. We may easily include a measurable dependence on the slow variable $\varepsilon k$ by assuming $g=g(x, k, z)$ is a Carathéodory function (this covers in particular the case $g=$ $g(x, z))$ and substitute the last sum in (6) by

$$
\sum_{k \in Z^{\varepsilon}(\Omega)} \varepsilon^{d} g\left(\varepsilon k, k, u_{k}\right) .
$$

Correspondingly, in Theorem 5.2, the integrand in the last term in (25) must be substituted by $\varphi\left(x, u^{1}, \ldots, u^{N}\right)$, where the definition of this last function is the same but taking $g(x, k, z)$ in place of $g(k, z)$ so that $x$ simply acts as a parameter.

The proof of Theorem 5.2 will be subdivided into a lower and an upper bound. Proof of the lower bound. Let $u^{\varepsilon} \rightarrow\left(u^{1}, \ldots, u^{N}\right)$ be such that $F_{\varepsilon}\left(u^{\varepsilon}\right) \leq c<+\infty$. Fixing $M \in \mathbb{N}$, we introduce the notation

$$
\begin{aligned}
J_{M}^{\varepsilon} & =\left\{z \in \mathbb{Z}^{d}: Q_{M}+z M \subset \frac{1}{\varepsilon} \Omega\right\}, \\
R^{\varepsilon} & =\mathcal{N}_{0}^{\varepsilon}(\Omega) \backslash \bigcup_{z \in J_{M}^{\varepsilon}} \mathcal{N}_{0}^{\varepsilon}\left(Q_{M}+z M\right), \\
S^{\varepsilon} & =Z^{\varepsilon}(\Omega) \backslash \bigcup_{z \in J_{M}^{\varepsilon}} Z\left(Q_{M}+z M\right)
\end{aligned}
$$

and write

where

$$
F_{\varepsilon}\left(u^{\varepsilon}\right)=\sum_{j=1}^{N} \mathrm{I}_{j}^{\varepsilon}+\mathrm{II}^{\varepsilon}+\mathrm{III}^{\varepsilon}+\mathrm{IV}^{\varepsilon}+\mathrm{V}^{\varepsilon},
$$

$$
\mathrm{I}_{j}^{\varepsilon}=\mathscr{F}_{\varepsilon}^{j}(u),
$$




$$
\begin{aligned}
\mathrm{II}^{\varepsilon} & =\sum_{j=1}^{N} \sum_{\left(k, k^{\prime}\right) \in \mathcal{N}_{j}^{\varepsilon}(\Omega) \backslash\left(C_{j} \times C_{j}\right)} \varepsilon^{d-1} a_{k k^{\prime}}\left(v_{k}-v_{k^{\prime}}\right)^{2}, \\
\mathrm{III}^{\varepsilon} & =\sum_{z \in J_{M}^{\varepsilon}} \varepsilon^{d}\left(\sum_{\left(k, k^{\prime}\right) \in \mathcal{N}_{0}^{\varepsilon}\left(Q_{M}+z M\right)} a_{k k^{\prime}}\left(v_{k}-v_{k^{\prime}}\right)^{2}+\sum_{k \in Z\left(Q_{M}+z M\right)} g\left(k, v_{k}\right)\right), \\
\mathrm{IV}^{\varepsilon} & =\sum_{\left(k, k^{\prime}\right) \in R^{\varepsilon}} \varepsilon^{d} a_{k k^{\prime}}\left(v_{k}-v_{k^{\prime}}\right)^{2}, \\
\mathrm{~V}^{\varepsilon} & =\sum_{k \in S^{\varepsilon}} \varepsilon^{d} g\left(k, v_{k}\right) .
\end{aligned}
$$

Note that

$$
\begin{aligned}
\mathrm{II}^{\varepsilon} & \geq 0, \\
\mathrm{IV}^{\varepsilon} & \geq-c / M+o(1), \\
V^{\varepsilon} & \geq-\max |g|\left(\left|\Omega \backslash \varepsilon^{d} \bigcup_{z \in J_{M}^{\varepsilon}}\left(Q_{M}+z M\right)\right|+o(1)\right),
\end{aligned}
$$

where we have taken into account that the interactions in $\mathrm{IV}^{\varepsilon}$ may be negative and

$$
\liminf _{\varepsilon \rightarrow 0} \sum_{j=1}^{N} \mathrm{I}_{j}^{\varepsilon} \geq \sum_{j=1}^{N} \liminf _{\varepsilon \rightarrow 0} \mathrm{I}_{j}^{\varepsilon} \geq \sum_{j=1}^{N} \int_{\Omega \cap \partial^{*}\left\{u^{j}=1\right\}} f_{\text {hom }}^{j}(v) d \mathscr{H}^{d-1} .
$$

It remains to estimate $\mathrm{III}^{\varepsilon}$. To that end, we introduce the set of indices $\Lambda_{M}^{\varepsilon}=\left\{z \in J_{M}^{\varepsilon}: u^{\varepsilon}\right.$ constant on every connected component of $A_{j} \cap\left(Q_{3 M}+z M\right)$, $j=1, \ldots, N\}$.

Note that

$$
\#\left(J_{M}^{\varepsilon} \backslash \Lambda_{M}^{\varepsilon}\right) \leq \frac{c_{M}}{\varepsilon^{d-1}}
$$

We then write

$$
\begin{aligned}
\mathrm{III}^{\varepsilon}= & \sum_{z \in \Lambda_{M}^{\varepsilon}} \varepsilon^{d}\left(\sum_{\left(k, k^{\prime}\right) \in \mathcal{N}_{0}^{\varepsilon}\left(Q_{M}+z M\right)} a_{k k^{\prime}}\left(v_{k}-v_{k^{\prime}}\right)^{2}+\sum_{k \in Z\left(Q_{M}+z M\right)} g\left(k, v_{k}\right)\right) \\
& +\sum_{z \in J_{M}^{\varepsilon} \backslash \Lambda_{M}^{\varepsilon}} \varepsilon^{d}\left(\sum_{\left(k, k^{\prime}\right) \in \mathcal{N}_{0}^{\varepsilon}\left(Q_{M}+z M\right)} a_{k k^{\prime}}\left(v_{k}-v_{k^{\prime}}\right)^{2}+\sum_{k \in Z\left(Q_{M}+z M\right)} g\left(k, v_{k}\right)\right) \\
\geq & \sum_{z \in \Lambda_{M}^{\varepsilon}} \varepsilon^{d} M^{d} \varphi_{M}\left(u_{1}^{\varepsilon}, \ldots, u_{N}^{\varepsilon}\right)-c \varepsilon^{d} M^{d} \max \left(|g|+\left|a_{k k^{\prime}}\right|\right) \#\left(J_{M}^{\varepsilon} \backslash \Lambda_{M}^{\varepsilon}\right),
\end{aligned}
$$

where $u_{j}^{\varepsilon}$ is the constant value taken by $u^{\varepsilon}$ on $\left(Q_{M}+z M\right) \cap C_{j}$. Here we suppose $M$ is large enough so that the connected component of $C_{j}$ containing $\left(Q_{M}+z M\right) \cap C_{j}$ 
is connected in $Q_{3 M}+z M$. We set

$$
U^{\varepsilon}=\sum_{z \in \Lambda_{M}^{\varepsilon}}\left(u_{1}^{\varepsilon}, \ldots, u_{N}^{\varepsilon}\right) \chi_{Q_{M}+z M}
$$

and $\varphi_{M}(0, \ldots, 0)=0$. Note that $U^{\varepsilon} \rightarrow U:=\left(u^{1}, \ldots, u^{N}\right)$ in $L^{1}(\Omega)^{N}$ so that

$$
\liminf _{\varepsilon \rightarrow 0} \mathrm{III}^{\varepsilon} \geq \liminf _{\varepsilon \rightarrow 0}\left(\int_{\Omega} \varphi_{M}\left(U^{\varepsilon}\right) d x-\varepsilon \max |g| c_{M} M^{d}\right)=\int_{\Omega} \varphi_{M}(U) d x
$$

by the Lebesgue dominated convergence theorem and the estimate (28).

Summing up the inequalities (26), (27), and (29), we get

$$
\liminf _{\varepsilon \rightarrow 0} F_{\varepsilon}\left(u^{\varepsilon}\right) \geq \sum_{j=1}^{N} \int_{\Omega \cap \partial^{*}\left\{u^{j}=1\right\}} f_{\text {hom }}^{j}(v) d \mathscr{H}^{d-1}+\int_{\Omega} \varphi_{M}(U) d x .
$$

The lower-bound inequality then follows by taking the limit as $M \rightarrow+\infty$, using Proposition 4.1 and the Lebesgue dominated convergence theorem.

Proof of the upper bound. We fix $U=\left(u^{1}, \ldots, u^{N}\right) \in \mathrm{BV}(\Omega ;\{1,-1\})^{N}$. For every $j=1, \ldots, N$, we choose $u^{j, \varepsilon} \rightarrow u^{j}$ a recovery sequence for $\mathscr{F}_{\text {hom }}^{j}\left(u^{j}\right)$. We tacitly extend all functions defined on $Z^{\varepsilon}(\Omega)$ to all of $\mathbb{Z}^{d}$ with the value +1 outside $Z^{\varepsilon}(\Omega)$. This does not affect the value of the energies but allows us to rigorously define some sets of indices $z$ in the sequel.

We fix $M \in \mathbb{N}$ large enough. As in Section 4, we introduce the sets of indices

$$
\tilde{J}_{M}^{\varepsilon}=\left\{z \in \mathbb{Z}^{d}:\left(Q_{M}+z M\right) \cap \frac{1}{\varepsilon} \Omega \neq \varnothing\right\},
$$

$\Lambda_{M}^{j, \varepsilon}=\left\{z \in J_{M}^{\varepsilon}: u^{\varepsilon}\right.$ constant on every connected component of $\left.A_{j} \cap\left(Q_{3 M}+z M\right)\right\}$

and give the estimate

$$
\sum_{j=1}^{N} \#\left(\tilde{J}_{M}^{\varepsilon} \backslash \Lambda_{M}^{j, \varepsilon}\right) \leq \frac{c_{M}}{\varepsilon^{d-1}} .
$$

Note that, if $z \in \bigcap_{j=1}^{N} \Lambda_{M}^{j, \varepsilon}$, then $u^{j, \varepsilon}=: u^{j, \varepsilon, z}$ is constant on $C_{j} \cap\left(Q_{M}+z M\right)$ for $j=1, \ldots, N$. Let $v^{\varepsilon, z}$ be a minimizer for $\widetilde{\varphi}_{M}\left(u^{1, \varepsilon, z}, \ldots, u^{N, \varepsilon, z}\right)$.

We define

$$
u_{k}^{\varepsilon}= \begin{cases}u_{k}^{j, \varepsilon} & \text { if } k \in C_{j}, j=1, \ldots, N, \\ v^{\varepsilon, z}(k-z M) & \text { if } k \in Q_{M}+z M \text { and } z \in \bigcap_{j=1}^{N} \Lambda_{M}^{j, \varepsilon}, \\ 1 & \text { otherwise. }\end{cases}
$$


We first estimate the energy on the strong connections. By the definition of $u^{j, \varepsilon}$, we have for all $j=1, \ldots, N$

$$
\lim _{\varepsilon \rightarrow 0} \sum_{\left(k, k^{\prime}\right) \in \mathcal{N}_{j}^{\varepsilon}(\Omega) \cap\left(C_{j} \times C_{j}\right)} \varepsilon^{d-1} a_{k k^{\prime}}\left(u_{k}^{\varepsilon}-u_{k^{\prime}}^{\varepsilon}\right)^{2}=\mathscr{F}_{\mathrm{hom}}^{j}\left(u^{j}\right)
$$

since $u^{\varepsilon}=u^{j, \varepsilon}$ on $C_{j}$. On the strong connections between points not in the infinite connected components $C_{j}$,

$$
\sum_{\left(k, k^{\prime}\right) \in \mathcal{N}_{j}^{\varepsilon}(\Omega) \backslash\left(C_{j} \times C_{j}\right)} \varepsilon^{d-1} a_{k k^{\prime}}\left(u_{k}^{\varepsilon}-u_{k^{\prime}}^{\varepsilon}\right)^{2}=0
$$

since $u^{\varepsilon}$ is constant on every connected component of $A_{j} \backslash C_{j}$. Note that here we have used the condition that $v^{\varepsilon, z}=1$ on $D_{M}$ in the definition of $\widetilde{\varphi}_{M}$.

We then examine the contribution due to the interaction between weak connections and the term $g$. We first look at the contributions on the cubes in the sets $\Lambda_{M}^{j, \varepsilon}$, where we can use the definition of $\widetilde{\varphi}_{M}$ : for every $z \in \bigcap_{j=1}^{N} \Lambda_{M}^{j, \varepsilon}$,

$$
\sum_{\left(k, k^{\prime}\right) \in \mathcal{N}_{0}^{\varepsilon}\left(Q_{M}+z M\right)} a_{k k^{\prime}}\left(u_{k}^{\varepsilon}-u_{k^{\prime}}^{\varepsilon}\right)^{2}+\sum_{k \in Z\left(Q_{M}+z M\right)} g\left(k, u_{k}^{\varepsilon}\right)=\widetilde{\varphi}_{M}\left(u^{1, \varepsilon, z}, \ldots, u^{N, \varepsilon, z}\right) .
$$

The contributions interior to all other cubes in $\tilde{J}_{M}^{\varepsilon}$ sum up to

$$
\begin{gathered}
\sum_{z \notin \bigcap_{j=1}^{N} \Lambda_{M}^{j, \varepsilon}} \varepsilon^{d}\left(\sum_{\left(k, k^{\prime}\right) \in \mathcal{N}_{0}^{\varepsilon}\left(Q_{M}+z M\right)} a_{k k^{\prime}}\left(u_{k}^{\varepsilon}-u_{k^{\prime}}^{\varepsilon}\right)^{2}+\sum_{k \in Z\left(Q_{M}+z M\right)} g\left(k, u_{k}^{\varepsilon}\right)\right) \\
\leq \varepsilon^{d} M^{d}\left(\# P_{0} \max a_{i l}+\max |g|\right) \sum_{j=1}^{N} \#\left(J_{M}^{\varepsilon} \backslash \Lambda_{M}^{j, \varepsilon}\right) \\
\leq \varepsilon M^{d} c_{M}^{\prime}+o(1)
\end{gathered}
$$

by (31) and the fact that the boundary of $\Omega$ has zero measure. Finally, the contribution due to the weak connection across the boundary of neighboring cubes is given by

$$
\begin{aligned}
& \sum \varepsilon^{d} \quad \sum \quad a_{k k^{\prime}}\left(u_{k}^{\varepsilon}-u_{k^{\prime}}^{\varepsilon}\right)^{2} \\
& z \neq z^{\prime} \in \bigcap_{j=1}^{N} \Lambda_{M}^{j, \varepsilon} \quad\left(k, k^{\prime}\right) \in \mathcal{N}_{0}^{\varepsilon}(\Omega), k \in Q_{M}+z M, k^{\prime} \in Q_{M}+z^{\prime} M \\
& \leq \varepsilon^{d} M^{d-1} \# J_{M}^{\varepsilon} \# P_{0} \max a_{i l} \leq \# P_{0} \max a_{i l} \frac{|\Omega|}{M} .
\end{aligned}
$$

From the inequalities above, we obtain

$$
\limsup _{\varepsilon \rightarrow 0} F_{\varepsilon}\left(u^{\varepsilon}\right) \leq \sum_{j=1}^{N} \mathscr{F}_{\text {hom }}^{j}\left(u^{j}\right)+\int_{\Omega} \widetilde{\varphi}_{M}\left(u^{1}, \ldots, u^{N}\right) d x+\# P_{0} \max a_{i l} \frac{|\Omega|}{M} .
$$

Letting $M \rightarrow+\infty$ and using Propositions 4.2 and 4.1 then gives the result. 


\section{Examples}

In the pictures in the following examples, weak connections are denoted by a dashed line and strong connections by a continuous line.

6.1. One-dimensional examples. In this section, we consider easy one-dimensional examples, highlighting the possibility of double-porosity behavior if long-range interactions are allowed, contrary to the continuum case. We use a slightly different notation than above, with the sums depending only on one index. The factor $\frac{1}{4}$ is just a normalization since $\left(u_{i}-u_{j}\right)^{2}$ is always a multiple of 4 .

Example 6.1 (weak inclusions on alternating lattice). Consider a system of weak nearest-neighbor interactions and strong next-to-nearest-neighbor interactions on the even-odd lattice (see figure below); namely,

$$
F_{\varepsilon}(u)=\frac{\beta}{4} \sum_{i=1}^{N_{\varepsilon}} \varepsilon\left(u_{i}-u_{i-1}\right)^{2}+\frac{\alpha}{4} \sum_{j=1}^{N_{\varepsilon} / 2-1}\left(u_{2 j+1}-u_{2 j-1}\right)^{2}+\sum_{i=1}^{N_{\varepsilon}} \varepsilon g\left(u_{i}\right),
$$

where we assume that $\Omega=[0,1]$ and $N_{\varepsilon}=1 / \varepsilon \in 2 \mathbb{N}$. In this case $N=1, A_{1}=$ $C_{1}=1+2 \mathbb{N}$, and $A_{0}=2 \mathbb{N}$.

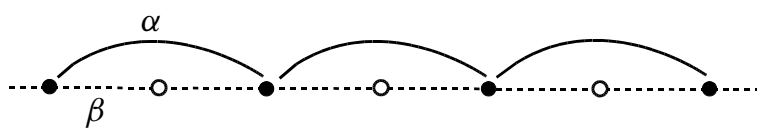

The $\Gamma$-limit is

$$
\begin{aligned}
F_{\mathrm{hom}}(u) & =\alpha \# S(u)+\frac{1}{2} \int_{0}^{1} g(u) d x+\frac{1}{2} \int_{0}^{1} \min \{g(u), g(-u)+2 \beta\} d x \\
& =\alpha \# S(u)+\int_{0}^{1} g(u) d x-\frac{1}{2} \int_{0}^{1} \max \{0, g(u)-g(-u)-2 \beta\} d x .
\end{aligned}
$$

The last term favors states with the same value on $A_{0}$ and $A_{1}$ if the integrand is 0 and of opposite sign if the integrand is positive. Note that this is always the case if we have a strong-enough "antiferromagnetic" nearest-neighbor interaction, i.e., $\beta$ is negative and $2|\beta|>|g(1)-g(-1)|$.

Example 6.2 (interacting sublattices). Consider a system of weak nearest-neighbor interactions and strong next-to-nearest-neighbor interactions:

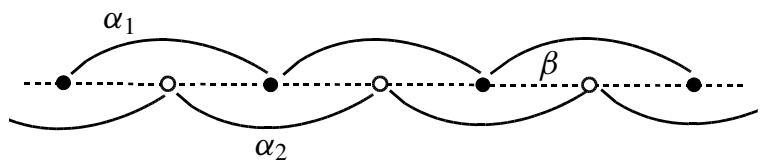


Here

$$
\begin{aligned}
F_{\varepsilon}(u)=\frac{\beta}{4} \sum_{i=1}^{N_{\varepsilon}} \varepsilon\left(u_{i}-u_{i-1}\right)^{2}+\frac{\alpha_{1}}{4} \sum_{j=1}^{N_{\varepsilon} / 2-1}\left(u_{2 j+1}-u_{2 j-1}\right)^{2} & \\
& +\frac{\alpha_{2}}{4} \sum_{j=0}^{N_{\varepsilon} / 2-1}\left(u_{2 j+2}-u_{2 j}\right)^{2}+\sum_{i=1}^{N_{\varepsilon}} \varepsilon g\left(u_{i}\right),
\end{aligned}
$$

where we assume that $N_{\varepsilon}=1 / \varepsilon \in 2 \mathbb{N}$. In this case, $N=2, A_{1}=C_{1} \stackrel{i=1}{=} 1+2 \mathbb{N}$, $A_{2}=C_{2}=2 \mathbb{N}$, and $A_{0}=\varnothing$.

The $\Gamma$-limit is

$$
\begin{aligned}
F_{\text {hom }}\left(u^{1}, u^{2}\right)=\alpha_{1} \# S\left(u^{1}\right) & +\alpha_{2} \# S\left(u^{2}\right) \\
& +\frac{1}{2} \int_{0}^{1} g\left(u^{1}\right) d x+\frac{1}{2} \int_{0}^{1} g\left(u^{2}\right) d x+\frac{\beta}{4} \int_{0}^{1}\left(u^{2}-u^{1}\right)^{2} .
\end{aligned}
$$

Note that, since $A_{0}=\varnothing$, we have no optimization in the interacting term, which then is just the pointwise limit of the nearest-neighbor interactions. Note moreover that in the case $\beta=0$ the interactions are completely decoupled.

Example 6.3 (interacting weak and strong sublattices). We consider the same pattern of interactions as in the previous example but with only strong connections on the odd lattice as in Example 6.1 (see figure below), i.e., with

$$
\begin{aligned}
F_{\varepsilon}(u)=\frac{\beta^{1}}{4} \sum_{i=1}^{N_{\varepsilon}} \varepsilon\left(u_{i}-u_{i-1}\right)^{2}+\frac{\beta^{2}}{4} \sum_{j=0}^{N_{\varepsilon} / 2-1} & \varepsilon\left(u_{2 j+2}-u_{2 j}\right)^{2} \\
& +\frac{\alpha}{4} \sum_{j=1}^{N_{\varepsilon} / 2-1}\left(u_{2 j+1}-u_{2 j-1}\right)^{2}+\sum_{i=1}^{N_{\varepsilon}} \varepsilon g\left(u_{i}\right) .
\end{aligned}
$$

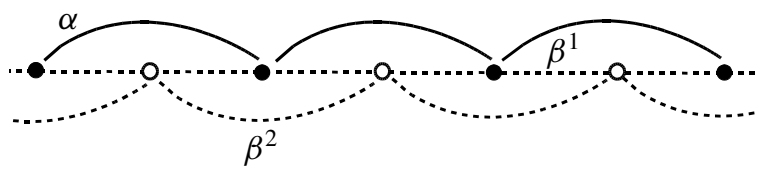

In this case, we have three possibilities:

- the minimizing values on the even lattice agree with those on the odd lattice (ferromagnetic overall behavior),

- the minimizing values on the even lattice disagree with those on the odd lattice (antiferromagnetic overall behavior), or

- the values on the even lattice alternate (antiferromagnetic behavior on the weak lattice).

The value of $\varphi$ is obtained by optimizing over these three possibilities; i.e.,

$$
\varphi(u)=\min \left\{g(u), \frac{g(u)+g(-u)}{2}+\beta^{1}, \frac{3 g(u)+g(-u)}{4}+\frac{\beta^{1}+\beta^{2}}{2}\right\},
$$


and we have

$$
F_{\text {hom }}(u)=\alpha \# S(u)+\int_{0}^{1} \varphi(u) d x .
$$

Example 6.4 (third-neighbor hard phases). In the system described in the figure below, involving strong third-neighbor interactions, we have two strong components and a $\Gamma$-limit obtained by minimization of the nearest and next-to-nearest neighbors. Using the same notation of the previous examples for the coefficients, we can write the limit as

$$
F_{\text {hom }}\left(u^{1}, u^{2}\right)=\alpha_{1} \# S\left(u^{1}\right)+\alpha_{2} \# S\left(u^{2}\right)+\int_{0}^{1} \varphi\left(u^{1}, u^{2}\right) d x,
$$

and

$$
\begin{aligned}
& \varphi\left(u^{1}, u^{2}\right)=\frac{1}{3}\left(g\left(u^{1}\right)+g\left(u^{2}\right)\right)+\frac{1}{4} \beta_{12}^{2}\left(u^{2}-u^{1}\right)^{2} \\
& \quad+\frac{1}{3} \min \left\{\frac{1}{4}\left(\left(\beta_{01}^{1}+\beta_{01}^{2}\right)\left(v-u^{1}\right)^{2}+\left(\beta_{02}^{1}+\beta_{02}^{2}\right)\left(v-u^{2}\right)^{2}\right)+g(v): v \in\{-1,1\}\right\} .
\end{aligned}
$$

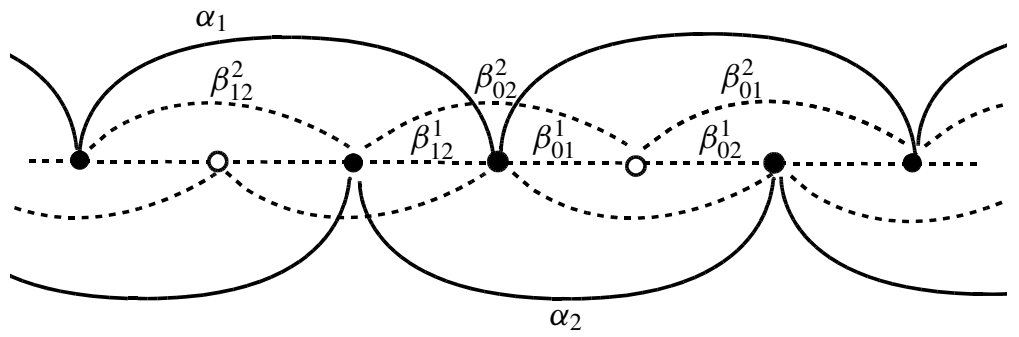

6.2. Higher-dimensional examples. In the following examples, we go back to the notation used in the statement of the main result. The normalization factor $\frac{1}{8}$ takes into account that each pair of nearest neighbors is accounted for twice.

Example 6.5 (a nearest-neighbor system with soft inclusions). Consider a nearestneighbor system in two dimensions in which $A_{0}=2 \mathbb{Z}^{2}$ and strong and weak interactions are given respectively by

$$
\frac{1}{8} \alpha\left(u_{k}-u_{k^{\prime}}\right)^{2}, \quad \frac{1}{8} \varepsilon \beta\left(u_{k}-u_{k^{\prime}}\right)^{2} .
$$

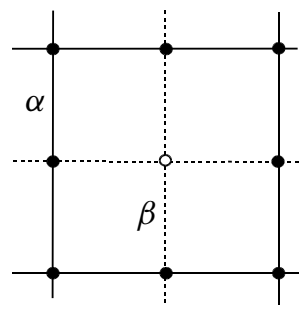


In this case,

$$
F_{\text {hom }}(u)=\frac{1}{2} \alpha \int_{S(u) \cap \Omega}\left\|v_{u}\right\|_{1} d \mathscr{H}^{1}+\int_{\Omega} \varphi(u) d x,
$$

where

$$
\varphi(u)=\min \left\{g(u), \frac{3 g(u)+g(-u)}{4}+\beta\right\} .
$$

Example 6.6 (a lattice with weak nearest-neighbor interactions). Consider strong interactions on a lattice of next-to-nearest neighbors as in the figure:

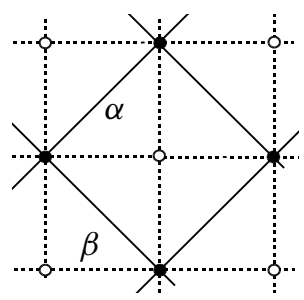

with weak nearest-neighbor interactions on the square lattice given respectively by

$$
\frac{1}{8} \alpha\left(u_{k}-u_{k^{\prime}}\right)^{2}, \quad \frac{1}{8} \varepsilon \beta\left(u_{k}-u_{k^{\prime}}\right)^{2},
$$

(the factor $\frac{1}{8}$ takes into account that each pair is accounted for twice). We only have one strong component, and with this choice of coefficients,

$$
F_{\text {hom }}(u)=\alpha \int_{\Omega \cap \partial\{u=1\}}\|v\|_{\infty} d \mathscr{H}^{1}+\int_{\Omega} \varphi(u) d x,
$$

where

$$
\varphi(u)=\min \left\{g(u), \frac{1}{2}(g(u)+g(-u))+\beta\right\} .
$$

Example 6.7. We include just the pictorial description of two more two-dimensional systems with a limit with two parameters (below, left) and with one limit parameter but with the possibility of an oscillating behavior on the weak lattice (below, right), analogous to the one-dimensional Examples 6.2 and 6.3, respectively.
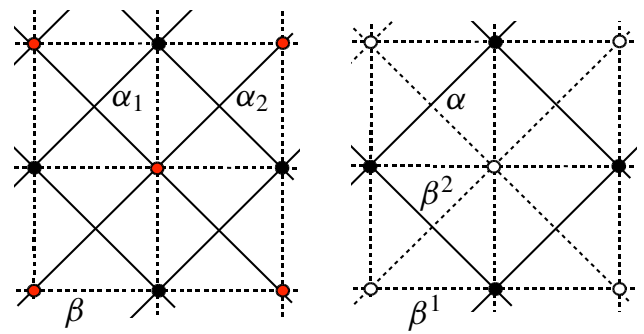

Example 6.8. We finally consider a three-dimensional two-periodic geometry, with one strong connected component pictured in Figure 3. Even in the absence of the 


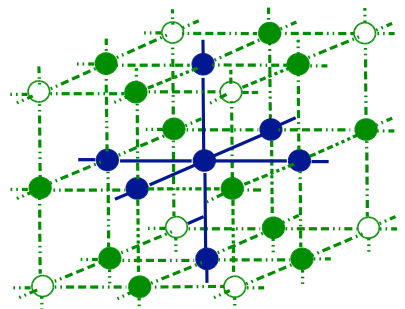

Figure 3. Oscillations in the infinite weak component.

forcing term $g$, we may have several competing microstructures in the determination of $\varphi$. In Figure 3, we have represented the uniform data $u=+1$ on the strong component with solid circles and a system of ferromagnetic connections between strong and weak sites (positive coefficients) and of antiferromagnetic connections between weak sites (a negative coefficient $\alpha$ ). Correspondingly, the minimal states have the value +1 on weak sites connected with the strong component (represented by solid circles) and the value -1 on the other sites (represented by white circles). Note that in this case the contribution of the weak phase is a constant.

\section{References}

[Alicandro and Gelli 2016] R. Alicandro and M. S. Gelli, "Local and nonlocal continuum limits of Ising-type energies for spin systems", SIAM J. Math. Anal. 48:2 (2016), 895-931.

[Amaziane and Pankratov 2016] B. Amaziane and L. Pankratov, "Homogenization of a model for water-gas flow through double-porosity media", Math. Methods Appl. Sci. 39:3 (2016), 425-451.

[Amaziane et al. 2006] B. Amaziane, L. Pankratov, and A. Piatnitski, "Homogenization of a class of quasilinear elliptic equations in high-contrast fissured media", Proc. Roy. Soc. Edinburgh (A) 136:6 (2006), 1131-1155.

[Amaziane et al. 2009a] B. Amaziane, L. Pankratov, and A. Piatnitski, "Nonlinear flow through double porosity media in variable exponent Sobolev spaces", Nonlinear Anal. Real World Appl. 10:4 (2009), 2521-2530.

[Amaziane et al. 2009b] B. Amaziane, L. Pankratov, and V. Rybalko, "On the homogenization of some double-porosity models with periodic thin structures", Appl. Anal. 88:10-11 (2009), 14691492 .

[Ambrosio et al. 2008] L. Ambrosio, N. Gigli, and G. Savaré, Gradient flows in metric spaces and in the space of probability measures, 2nd ed., Birkhäuser, Basel, 2008.

[Arbogast et al. 1990] T. Arbogast, J. Douglas, Jr., and U. Hornung, "Derivation of the double porosity model of single phase flow via homogenization theory", SIAM J. Math. Anal. 21:4 (1990), 823-836.

[Barenblatt et al. 1960] G. I. Barenblatt, Iu. P. Zheltov, and I. N. Kochina, "Basic concepts in the theory of seepage of homogeneous liquids in fissured rocks [strata]", J. Appl. Math. Mech. 24:5 (1960), 1286-1303. 
[Bourgeat et al. 1996] A. Bourgeat, S. Luckhaus, and A. Mikelić, "Convergence of the homogenization process for a double-porosity model of immiscible two-phase flow", SIAM J. Math. Anal. 27:6 (1996), 1520-1543.

[Bourgeat et al. 1998] A. Bourgeat, A. Mikelić, and A. Piatnitski, "Modèle de double porosité aléatoire", C. R. Acad. (I) 327:1 (1998), 99-104.

[Bourgeat et al. 1999] A. Bourgeat, M. Goncharenko, M. Panfilov, and L. Pankratov, "A general double porosity model", C. R. Acad. (IIB) 327:12 (1999), 1245-1250.

[Bourgeat et al. 2003] A. Bourgeat, G. A. Chechkin, and A. L. Piatnitski, "Singular double porosity model”, Appl. Anal. 82:2 (2003), 103-116.

[Braides 1998] A. Braides, Approximation of free-discontinuity problems, Lecture Notes in Mathematics 1694, Springer, Berlin, 1998.

[Braides 2002] A. Braides, $\Gamma$-convergence for beginners, Oxford Lecture Series in Mathematics and its Applications 22, Oxford University Press, Oxford, 2002.

[Braides 2006] A. Braides, "A handbook of $\Gamma$-convergence", pp. 101-213 in Handbook of differential equations: stationary partial differential equations, vol. 3, ch. 2, edited by M. Chipot and P. Quittner, Elsevier, Amsterdam, 2006.

[Braides 2014a] A. Braides, "Discrete-to-continuum variational methods for Lattice systems", pp. 997-1015 in Proceedings of the International Congress of Mathematicians (Seoul, 2014), vol. IV, edited by S. Y. Jang et al., Kyung Moon Sa, Seoul, 2014.

[Braides 2014b] A. Braides, Local minimization, variational evolution and $\Gamma$-convergence, Lecture Notes in Mathematics 2094, Springer, Cham, 2014.

[Braides and Piatnitski 2013] A. Braides and A. Piatnitski, "Homogenization of surface and length energies for spin systems", J. Funct. Anal. 264:6 (2013), 1296-1328.

[Braides and Solci 2013] A. Braides and M. Solci, "Multi-scale free-discontinuity problems with soft inclusions", Boll. Unione Mat. Ital. (9) 6:1 (2013), 29-51.

[Braides and Solci 2015] A. Braides and M. Solci, "Motion of discrete interfaces through mushy layers", preprint, 2015. To appear in J. Nonlinear Sci. arXiv 1506.04062v1

[Braides et al. 2004] A. Braides, V. Chiadò Piat, and A. Piatnitski, "A variational approach to doubleporosity problems", Asymptot. Anal. 39:3-4 (2004), 281-308.

[Braides et al. 2015] A. Braides, V. Chiadò Piat, and A. Piatnitski, "Homogenization of discrete high-contrast energies", SIAM J. Math. Anal. 47:4 (2015), 3064-3091.

[Caffarelli and de la Llave 2005] L. A. Caffarelli and R. de la Llave, "Interfaces of ground states in Ising models with periodic coefficients”, J. Stat. Phys. 118:3-4 (2005), 687-719.

[Cherdantsev and Cherednichenko 2012] M. Cherdantsev and K. D. Cherednichenko, "Two-scale $\Gamma$ convergence of integral functionals and its application to homogenisation of nonlinear high-contrast periodic composites", Arch. Ration. Mech. Anal. 204:2 (2012), 445-478.

[Choquet 2004] C. Choquet, "Derivation of the double porosity model of a compressible miscible displacement in naturally fractured reservoirs", Appl. Anal. 83:5 (2004), 477-499.

[Choquet and Pankratov 2010] C. Choquet and L. Pankratov, "Homogenization of a class of quasilinear elliptic equations with non-standard growth in high-contrast media", Proc. Roy. Soc. Edinburgh (A) 140:3 (2010), 495-539.

[Hornung 1997] U. Hornung (editor), Homogenization and porous media, Interdisciplinary Applied Mathematics 6, Springer, New York, 1997.

[Konyukhov and Pankratov 2015] A. Konyukhov and L. Pankratov, "Upscaling of an immiscible non-equilibrium two-phase flow in double porosity media", Appl. Anal (online publication July 2015). 
[Marchenko and Khruslov 2006] V. A. Marchenko and E. Ya. Khruslov, Homogenization of partial differential equations, Progress in Mathematical Physics 46, Birkhäuser, Boston, 2006.

[Panasenko 1991] G. P. Panasenko, "Multicomponent homogenization of processes in strongly nonhomogeneous structures", Sb. Math. 69:1 (1991), 143-153.

[Panfilov 2000] M. Panfilov, Macroscale models of flow through highly heterogeneous porous media, Theory and Applications of Transport in Porous Media 16, Springer, Dordrecht, 2000.

[Pankratov and Piatnitski 2002] L. Pankratov and A. Piatnitski, "Nonlinear 'double porosity' type model", C. R. Math. 334:5 (2002), 435-440.

[Pankratov and Rybalko 2003] L. S. Pankratov and V. A. Rybalko, "Asymptotic analysis of a double porosity model with thin fissures", Sb. Math. 194:1 (2003), 123-150.

[Pankratov et al. 2003] L. Pankratov, A. Piatnitskii, and V. Rybalko, "Homogenized model of reactiondiffusion in a porous medium", C. R. Mécanique 331:4 (2003), 253-258.

[Sandrakov 1999a] G. V. Sandrakov, "Homogenization of elasticity equations with contrasting coefficients", Sb. Math. 190:12 (1999), 1749-1806.

[Sandrakov 1999b] G. V. Sandrakov, "Homogenization of parabolic equations with contrasting coefficients", Izv. Math. 63:5 (1999), 1015-1061.

[Seppecher et al. 2011] P. Seppecher, J.-J. Alibert, and F. Dell Isola, "Linear elastic trusses leading to continua with exotic mechanical interactions”, J. Phys. Conf. Ser. 319 (2011), 012018.

[Solci 2009] M. Solci, "Double-porosity homogenization for perimeter functionals", Math. Methods Appl. Sci. 32:15 (2009), 1971-2002.

[Solci 2012] M. Solci, "Multiphase double-porosity homogenization for perimeter functionals", Math. Methods Appl. Sci. 35:5 (2012), 598-620.

[Yeh 2006] L.-M. Yeh, "Homogenization of two-phase flow in fractured media", Math. Models Methods Appl. Sci. 16:10 (2006), 1627-1651.

Received 1 Dec 2015. Revised 21 Jan 2016. Accepted 28 Feb 2016.

ANDREA BRAIDES: braides@mat.uniroma2.it

Dipartimento di Matematica, Università di Roma Tor Vergata, via della ricerca scientifica 1, I-00133 Roma, Italy

VALERIA CHIADÒ PIAT: d001854@polito.it

Dipartimento di Matematica, Politecnico di Torino, corso Duca degli Abruzzi 24, I-10129 Torino, Italy

MARGHERITA SOLCI: margherita@uniss.it

Dipartimento di Architettura, Design, Urbanistica, Università di Sassari, piazza Duomo 6, I-07041 Alghero, Italy 
EDITORIAL BOARD

ANTONIO CARCATERRA

ERIC A. CARLEN

FRANCESCO DELL'ISOLA

RAFFAELE ESPOSITO

ALBERT FANNJIANG

Gilles A. FranCFORT

Pierangelo MARCATI

JEAN-JACQUES MARIGO

PETER A. MARKOWICH

MARTIN OSTOJA-STARZEWSKI

PIERRE SEPPECHER

DAVID J. STEIGMANN

PAUl STEINMANN

PierRe M. SuQueT

MANAGING EDITORS

MICOL AMAR

CORRADO LATTANZIO

ANGELA MADEO

MARTIN OSTOJA-STARZEWSKI

ADVISORY BOARD

ADNAN AKAY

Holm AltenBaCH

MICOL AMAR

HARM ASKES

TEODOR ATANACKOVIĆ

VICTOR BERDICHEVSKY

GUY BOUCHITTÉ

ANDREA BRAIDES

ROBERTO CAMASSA

MAURO CARFORE

ERIC DARVE

FELIX DARVE

ANNA DE MASI

GianPiEtro DEL Piero

EMMANUELE Di BENEDETTO

BERNOLD FIEDLER

IRENE M. GAMBA

DAVID Y. GAO

SERGEY GAVRILYUK

TIMOTHY J. HEALEY

DOMINIQUE JEULIN

ROGER E. KHAYAT

CORRADO LATTANZIO

ROBERT P. LIPTON

ANGELO LUONGO

ANGELA MADEO

JUAN J. MANFREDI

CARLO MARCHIORO

GÉRARD A. MAUGIN

ROBERTO NATALINI PATRIZIO NEFF

ANDREY PIATNITSKI

ERRICO PRESUTTI

MARIO PULVIRENTI

LUCIO RUSSO

Miguel A. F. SANJUAN

PATRICK SElVADURAI

ALEXANDER P. SEYRANIAN

MIROSLAV ŠILHAVÝ

GUIDO SWEERS

ANTOINETTE TORDESILLAS

LEV TRUSKINOVSKY

JUAN J. L. VELÁZQUEZ VINCENZO VESPRI ANGELO VULPIANI msp.org/memocs

Università di Roma "La Sapienza", Italia

Rutgers University, USA

(CO-CHAIR) Università di Roma "La Sapienza", Italia

(TREASURER) Università dell'Aquila, Italia

University of California at Davis, USA

(CO-CHAIR) Université Paris-Nord, France

Università dell'Aquila, Italy

École Polytechnique, France

DAMTP Cambridge, UK, and University of Vienna, Austria

(CHAIR MANAGING EDITOR) Univ. of Illinois at Urbana-Champaign, USA

Université du Sud Toulon-Var, France

University of California at Berkeley, USA

Universität Erlangen-Nürnberg, Germany

LMA CNRS Marseille, France

Università di Roma "La Sapienza", Italia

Università dell'Aquila, Italy

Université de Lyon-INSA (Institut National des Sciences Appliquées), France

(CHAIR MANAGING EDITOR) Univ. of Illinois at Urbana-Champaign, USA

Carnegie Mellon University, USA, and Bilkent University, Turkey

Otto-von-Guericke-Universität Magdeburg, Germany

Università di Roma "La Sapienza", Italia

University of Sheffield, UK

University of Novi Sad, Serbia

Wayne State University, USA

Université du Sud Toulon-Var, France

Università di Roma Tor Vergata, Italia

University of North Carolina at Chapel Hill, USA

Università di Pavia, Italia

Stanford University, USA

Institut Polytechnique de Grenoble, France

Università dell'Aquila, Italia

Università di Ferrara and International Research Center MEMOCS, Italia

Vanderbilt University, USA

Freie Universität Berlin, Germany

University of Texas at Austin, USA

Federation University and Australian National University, Australia

Université Aix-Marseille, France

Cornell University, USA

École des Mines, France

University of Western Ontario, Canada

Università dell' Aquila, Italy

Louisiana State University, USA

Università dell'Aquila, Italia

Université de Lyon-INSA (Institut National des Sciences Appliquées), France University of Pittsburgh, USA

Università di Roma "La Sapienza”, Italia

Université Paris VI, France

Istituto per le Applicazioni del Calcolo "M. Picone", Italy

Universität Duisburg-Essen, Germany

Narvik University College, Norway, Russia

Università di Roma Tor Vergata, Italy

Università di Roma "La Sapienza”, Italia

Università di Roma “Tor Vergata”, Italia

Universidad Rey Juan Carlos, Madrid, Spain

McGill University, Canada

Moscow State Lomonosov University, Russia

Academy of Sciences of the Czech Republic

Universität zu Köln, Germany

University of Melbourne, Australia

École Polytechnique, France

Bonn University, Germany

Università di Firenze, Italia

Università di Roma La Sapienza, Italia

MEMOCS (ISSN 2325-3444 electronic, 2326-7186 printed) is a journal of the International Research Center for the Mathematics and Mechanics of Complex Systems at the Università dell'Aquila, Italy.

Cover image: "Tangle” by $\odot$ John Horigan; produced using the Context Free program (contextfreeart.org).

PUBLISHED BY

7 mathematical sciences publishers

nonprofit scientific publishing

http://msp.org/

(C) 2016 Mathematical Sciences Publishers 
Mathematics and Mechanics of Complex Systems vol. 4 no. 1

Gradient materials with internal constraints

Albrecht Bertram and Rainer Glüge

Unified geometric formulation of material uniformity and evolution

Marcelo Epstein and Manuel de León

Electromechanics of polarized lipid bilayers

David J. Steigmann and Ashutosh Agrawal

Orthogonal polynomials and Riesz bases applied to the solution of Love's equation

Pierluigi Vellucci and Alberto Maria Bersani

Modeling capillary hysteresis in unsatured porous media

Gérard Gagneux and Olivier Millet

Discrete double-porosity models for spin systems

Andrea Braides, Valeria Chiadò Piat and Margherita Solci

Correction to "On the theory of diffusion and swelling in finitely deforming elastomers"

Gary J. Templet and David J. Steigmann

MEMOCS is a journal of the International Research Center for the Mathematics and Mechanics of Complex Systems at the Università dell' Aquila, Italy.

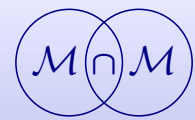

